Fabian Cremer, Silvia Daniel, Marina Lemaire, Katrin Moeller, Matthias Razum, Arnošt Štanzel

\title{
Data meets history: A research data management strategy for the historically oriented humanities
}

\begin{abstract}
The growing relevance of research data to practices, methodology and policies poses challenges for the historically oriented humanities in developing their own concept of research data management. A broader definition of research data is derived from recent discussions and an examination of the historical research workflow reveals its ongoing digital transformation. Based on current developments in Germany, this article provides an outline for a strategic approach towards a domain-specific research data management strategy including applicable metadata concepts, cultural conditions for data sharing and initial suggestions regarding the specification of the FAIR principles for the historically oriented humanities.
\end{abstract}

\section{Introduction}

The ubiquitous digital turn is affecting all relevant aspects of society, including research. It is also shaping the future of the historically oriented humanities on many fronts: 1) its methodology, processes and practices, which are being transformed and extended through digital technologies; 2) its research objects, which are more and more either digitised or born-digital and therefore dependent on digital technologies; 3 ) its organisations and policies, which demand an examination of - and more often an adaption to - digital technologies. The ever-growing amount of digital data being gathered and produced in the historically oriented humanities raises questions of how this data can be collected, analysed, stored, archived, catalogued and made available for reuse - in short: how data can be effectively managed and its quality assured. Thus far, the development of research data management (RDM) has been fuelled primarily by funders and research organisations through the definition of policies and by libraries and computing centres through investments in information infrastructures. However, the process of data collection has become a highly subject-specific, competencebased process and, as a consequence, research communities have recently been called upon to actively participate in these developments and to formulate their 
own principles in cooperation with the information infrastructures mentioned above and with memory institutions. ${ }^{1}$

In Germany, which serves as the use case for our considerations in this article, the Council for Scientific Information Infrastructures (RfII) formulated recommendations for how research data should be managed in the future within the national research landscape. ${ }^{2}$ Subsequently, the Federal Government initiated a National Research Data Infrastructure (NFDI). It is to be implemented by thirty consortia, each representing a research community with specific requirements, including a consortium for the historically oriented humanities (4Memory). ${ }^{3}$ The consortia assess the needs deriving from the digital research methods, practices and data within their domain with the goal of developing researchdriven and domain-oriented standards and services.

The internationally accepted primary guidelines for scientific data management and stewardship are the "FAIR principles", ${ }^{4}$ which aim to improve the Findability, Accessibility, Interoperability, and Reuse of research data. However, as FAIR addresses all disciplines, the principles are by nature generic. Their implementation into practice and the development of complementary quality standards for research data require a disciplinary approach. In the following sections, we will discuss an approach for the historically oriented humanities.

\footnotetext{
1 The German Research Foundation (DFG) is appealing to the scientific communities to establish discipline-specific regulations for the handling of research data and to establish procedures for the recognition of achievements in the publication of research data. A few disciplines have already drawn up discipline-specific guidelines. https://web.archive.org/web/ 20201022005933/https://www.dfg.de/en/research_funding/proposal_review_decision/appli cants/research_data/index.html (2020-20-11).

2 German Council for Scientific Information Infrastructures, Enhancing Research Data Management: Performance through Diversity. Recommendations regarding structures, processes, and financing for research data management in Germany, Göttingen 2016: urn:nbn:de:101:1-20161214992.

3 For the aims and task areas of the 4Memory application within the NFDI see: 4memory.de. This article is based on discussions within the NFDI4Memory consortium. As a consequence, this articles gives an overview and references several papers published from the German research community. We thank our 4Memory colleagues for their input and comments and especially John C. Wood for his critical review and editing.

4 Mark D. Wilkinson et al., 'The FAIR Guiding Principles for Scientific Data Management and Stewardship', in: Scientific Data, 3/1 (2016), https://doi.org/10.1038/sdata.2016.18. A closer look at the requirements and some examples for the different tasks for researchers and providers are given in: Angelina Kraft, 'The FAIR Data Principles for Research Data', in: TIB-Blog, 2017, https:// blogs.tib.eu/wp/tib/2017/09/12/the-fair-data-principles-for-research-data/ (2020-11-20).
} 


\section{The definitions of research data}

Historians have always been data reusers, creators and curators. Their research data consist of historical records that are sought out, gathered, validated, annotated, compared and represented in contextualised formats in order to reconstruct the history of mankind. They use their research data to answer specific research questions and to support statements, methods, arguments, hypotheses or theories. Research-driven data aggregation thus creates data collections reflecting particular interests and methods. These collections contain research data of various provenance - from memory institutions, government agencies, private persons and companies, citizen-science organisations, and many others, because everything left by people in the past can help to answer a research question. For this reason, the range of primary research data (historical sources) ranges from all types of textual and numerical documents to material objects from art, culture, science and everyday life to oral tradition and memories, only a few of which have been digitised professionally or in standardised ways.

Thus far, historians have yet to reach a consensus on the definition of "research data" in historical research. Nonetheless, they have traditionally distinguished between the sources from which they extract historical knowledge and the information they create through research that is guided by particular questions, methodological assumptions, processes and perspectives. With the emergence of debates on research data in the last two decades, many within the historically oriented humanities have started to describe the historical record itself - i.e. the sources of historical research - as "research data". While the definition of historical sources as "research data" is not without controversy, it has been tentatively established that it includes digital representations of historical records as fitting subjects for data management. In essence, the concept "data" now encompasses all forms of representation, from the analogue object to the reused data produced during research. During this process historians traditionally create source excerpts, transcriptions, bibliographical and biographical records, finding aids, inventories, interviews, statistical data (e.g. time series), and/or structured information on events, facts, places or persons, including the provenance and context of any of this information in order to correlate the different elements of these data.

5 See surveys in Germany: Peter Andorfer, 'Forschungsdaten in den (digitalen) Geisteswissenschaften. Versuch einer Konkretisierung', DARIAH-DE Working Papers 14 (2015), urn:nbn:de: gbv:7-dariah-2015-7-2, here 13-14; Boris Queckbörner, 'Forschungsdaten und Forschungsdatenmanagement in der Geschichtswissenschaft’, 2019, https://doi.org/10.18452/20460, here 31. 
The RfII states that research data is "any data that is generated in the course of scientific work, for example through observations, experiments, simulations, surveys, questioning, source analysis, records, digitalisation, evaluations” and includes "such data which are not acquired by science itself, but which science accesses for research purposes in order to use them as a methodologically necessary basis for the concrete research process". ${ }^{6}$ Adapting this concept to historical scholarship adds new types of data to the accepted categories of historical sources and published results, leading to a broader definition of research data, as the position paper of the German Historical Association (VHD) on the NFDI elaborates: ${ }^{7}$

- direct digital representation of cultural objects (digital copies)

- measurement, survey and survey data (e.g. from empirical research)

- enriched, expanded, annotated, commentated, pre-processed forms of representation

- critical editions of sources and texts

- question-directed data collections and databases

- specialised knowledge bases, bibliographies, taxonomies, ontologies, controlled vocabularies, authority data

- algorithms and specialised software tools

- specialist applications such as simulations

- dynamic or static visualisations

- traditional as well as new forms of presenting results (e.g. blog series, complex narratives with multimedia and hypertext elements)

- digital research environments

- specialised material collections, portals

- subject-specific research tools

This wider understanding leads to a general definition of research data in the historically oriented disciplines: any information, regardless of its provenance, is considered "research data" as soon as it is collected, described, annotated, evaluated and/or created and stored in machine-readable form for the purpose of maintaining the traceability of research results or for archiving, citation and further processing. Hence, research data include all media formats and digital

6 RfII, The Data Quality Challenge. Recommendations for Sustainable Research in the Digital Turn, RfII - German Council for Scientific Information Infrastructures, Göttingen, 2020, http:// www.rfii.de/?wpdmdl=4203 (2020-11-20), here 106.

7 VHD, 'Positionspapier des Verbandes der Historiker und Historikerinnen Deutschlands (VHD) zur Schaffung nationaler Forschungsdateninfrastrukturen (NFDI)’, 2017, https://web.archive.org/ web/20200807014629/https://www.historikerverband.de/verband/stellungnahmen/positionspap ier-zur-schaffung-nationaler-forschungsdateninfrastrukturen-nfdi.html, here 3-4. 
representations of analogue sources as well as any information that can be used to answer research questions. ${ }^{8}$

The considerations and processes mentioned above are common to other fields in the humanities, which share generic research data management needs. ${ }^{9}$ Research data in the humanities is not "generated" in a specific setting but rather gathered and captured - in a process guided by specific aims - as an act of creation and construction. ${ }^{10}$ Potential sources are endless, since, for a humanities scholar, any legacy of human activity or experience is potentially a data source. ${ }^{11}$ However, historical data pose specific challenges to the historical method in digital scholarship, which have to be addressed collaboratively in the research communities.

First, the incompleteness of the historical record and its separation from its original context makes establishing the authenticity of sources challenging. Digitisation often adds another layer of abstraction and complexity, as digital representations lack the physical attributes normally used to prove authenticity or, even more importantly, to question it.

Second, data collections combine layers of complexity: their sources vary in format and structure, with multimedia collections and unstructured items being the norm: their human-created contents are ambiguous and often contradictory.

Third, establishing data as a legitimate research output requires contextualising information regarding its historical origin, its provenance through data processing and its form and intention of representation. Larger collections and digitised formats have often instead encouraged decontextualisation. Finally, analysing born-digital data as historical sources requires an extension of source

8 Jenny Oltersdorf and Stefan Schmunk, 'Von Forschungsdaten und wissenschaftlichen Sammlungen', in: Bibliothek Forschung und Praxis, 40/2 (2016), 179-185, https://doi.org/10.1515/bfp2016-0036.

9 The characteristics of humanities data are explored in: Christof Schöch, 'Big? Smart? Clean? Messy? Data in the Humanities', in: Journal of Digital Humanities, 2/3 (2013), 2-13, http://jour nalofdigitalhumanities.org/2-3/big-smart-clean-messy-data-in-the-humanities/ (2020-11-20); A formal data lifecycle is suggested in: Johanna Puhl et al., 'Diskussion und Definition eines Research Data LifeCycle für die digitalen Geisteswissenschaften', DARIAH-DE Working Papers, 11 (2015), urn:nbn:de:gbv:7-dariah-2015-4-4; The tensions between conception and implementation of research data in the humanities are discussed in: Fabian Cremer, Lisa Klaffki and Timo Steyer, 'Der Chimäre auf der Spur: Forschungsdaten in den Geisteswissenschaften', in: $o$-bib, 5.2 (2018), 142-62, https://doi.org/10.5282/o-bib/2018H2S142-162.

10 Johanna Drucker is elaborating on the concept of data as capta, taken and constructed: Johanna Drucker, 'Humanities Approaches to Graphical Display', in: Digital Humanities Quarterly, 005/1 (2011), http://www.digitalhumanities.org/dhq/vol/5/1/000091/000091.html (2020-11-20).

11 Christine L. Borgman, Scholarship in the Digital Age: Information, Infrastructure, and the Internet, Cambridge 2007, here 216. 
criticism - from production, provenance and context to the different representations of data - reflecting the "dual nature of digital data as both content and form". ${ }^{12}$ The development of historical methods on data and their critical reflection will significantly change the historical-scientific culture of the discipline, enable different research questions, expand the canon of methods, and can possibly change or even reshape previous knowledge orders. ${ }^{13}$

\section{The workflows of historical research}

Historical research ranges from individual research - still its most common setting - or research groups in smaller projects or institutions to large, collaborative and interdisciplinary projects. In the traditional workflow, researchers collect their sources (often only available in analogue form) in archives and libraries; analyse the selected corpus and answer their research questions through long-established historical methods. The resulting publication, usually a printed book or peerreviewed journal article, explains the selection criteria, interprets the sources and cites their (physical) location, while an attached list of sources provides an overview of the collections used (figure 1).

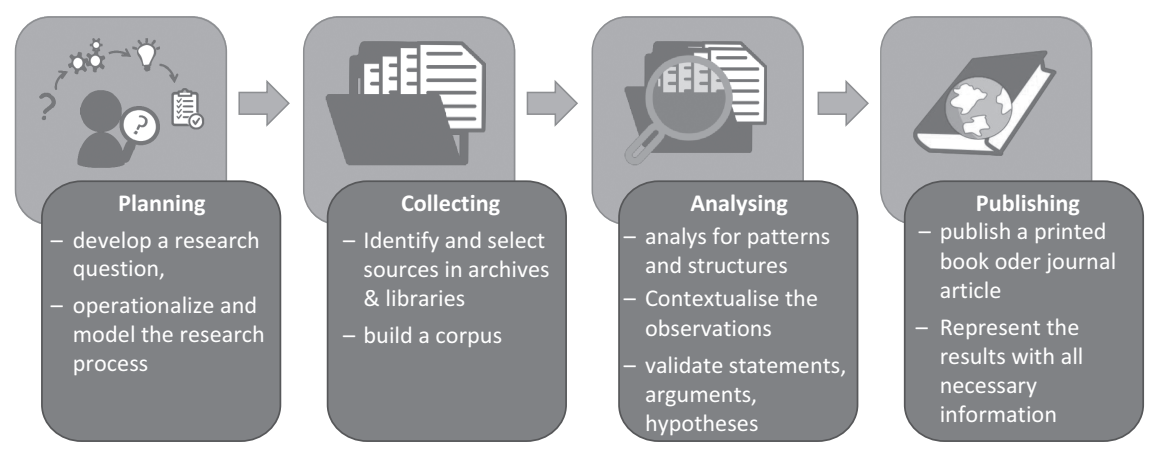

Figure 1: Example of an analogue historical research workflow.

12 Christine Barats, Valérie Schafer, and Andreas Fickers, 'Fading Away . . . The Challenge of Sustainability in Digital Studies', in: Digital Humanities Quarterly, 14/3 (2020), http://www.digi talhumanities.org/dhq/vol/14/3/000484/000484.html (2020-11-20), here 18.

13 See Andreas Fickers, 'Update für die Hermeneutik. Geschichtswissenschaft auf dem Weg zur digitalen Forensik?’, in: Zeithistorische Forschungen, 17/1 (2020), 157-68, https://doi.org/ 10.14765/ZZF.DOK-1765. 
While this standardised approach guarantees transparency and intellectual comprehensibility, it limits the reuse of data to the interpretations and references made therein; moreover, the documentation, contextualisation, and enrichment of the sources is not transferred to the memory institutions that possess the original sources. Since the individual project is still the norm in the humanities, a research data management strategy must include measures to a) enrich analogue workflows with digital methods, services and tools; b) advance existing community practices towards standardised data management practices; and c) develop channels and opportunities to integrate such essential practices into the whole community.

Another notable scenario would be a traditionally oriented but completely digital project that could be described as follows. First, historically oriented researchers work together with an archive to digitise the original sources, enrich them with basic metadata, make them available as FAIR data and guarantee the long-term preservation of the digitised material, ensuring the quality of the digital objects according to the funder's guidelines. Next, the researchers go through the digitised material, augment it, correct the metadata, transcribe the sources and annotate the text with keywords representing the persons, institutions, places and events referred to therein with software tools provided by the digital research support centre. Based on this data, digital methods and tools for analysis will be applied to answer the research questions. Further, the project team designs data representation layers (which include an API and data dumps) in addition to a website and browser-based search and discovery functionalities. Finally, the research results (data and services) are hosted and maintained by an IT-infrastructure service institution, with data discovery being managed by information professionals from the memory institution. An institutional research data management service centre accompanies the project, serving as stewards for proper research data management, as described in the project's data management plan (figure 2).

Over the last decade an increasing number of projects have digitised or digitally transformed parts of their workflows. The scenario described above is still largely a vision of the future: such projects are extremely rare, as they provide multiple challenges for traditionally trained and skilled researchers, which additionally face the problem that collaborations among researchers, memory institutions and infrastructure partners are mostly insufficient. ${ }^{14}$ To advance the

14 See the different discussions in: Historische Grundwissenschaften und die digitale Herausforderung, ed. by Rüdiger Hohls, Claudia Prinz and Eva Schlotheuber, Historisches Forum (Berlin, 2016), XVIII, https://doi.org/10.18452/18771; and the introduction in: Marina Lemaire et al., Das DIAMANT-Modell 2.0. Modellierung des FDM-Referenzprozesses und Empfehlungen für die 


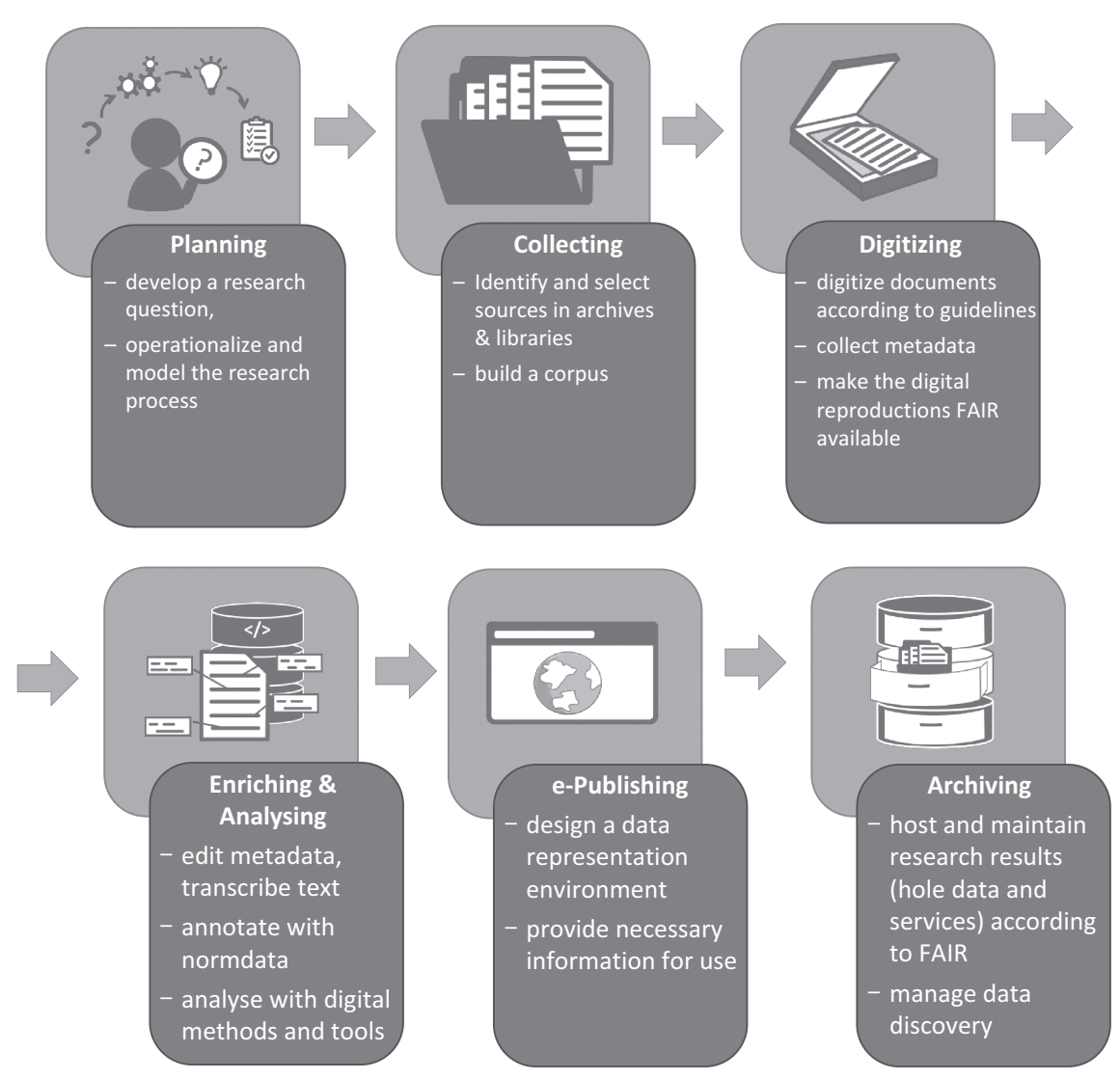

Figure 2: Example of a digital historical research workflow.

digital transformation of research, a research data management strategy must aim to: a) promote the implementation of digital workflows which enhance quality and sustainability of the research data; b) foster data management skills as a core competence in the historical sciences and the research supporting facilities such as data centers, libraries and RDM service units; and c) increase interoperability of the software tools and information systems used in order to create a consistent framework on project level and a functional ecosystem within the research domain.

Implementierung einer institutionellen FDM-Servicelandschaft, eSciences Working Papers 05, Trier 2020, https://doi.org/10.25353/ubtr-xxxx-f5d2-fffb, here 6-7. 
While improving traditional research workflows, the field of "digital history" seeks to enhance and expand historical methodologies by integrating quantitative or computational methods such as text mining or network analysis. ${ }^{15}$ These methods also add simulations, visualisations and research software to the research data types in the historical research domain. Understood as a transitional term, "digital history” emphasises a shift in research practices toward addressing born-digital data as a new type of source, developing digital methods for analysis and establishing new forms of academic publishing. ${ }^{16}$ Using digital data, tools and methods causes dependencies on information technology and vice versa, which has to be incorporated into the principles of historical knowledge production as "digital hermeneutics". ${ }^{17}$ Thus, it is crucial that a research data management strategy promotes a continuous dialogue in the historically oriented humanities on a) digital methods and their application in the different fields; b) on bringing data literacy into historical methodology and information science methodologies; and c) on the conditions of digital knowledge production, including its ethical and legal implications.

Historical research, regardless of its degree of digital transformation, is defined by its techniques. The historically oriented humanities share scholarly principles with the humanities overall, but domain-specific aspects are pointed out in the white paper "Digital History and Argument" of the Roy Rosenzweig Center for History and New Media: ${ }^{18}$

15 An introduction in methods in digital history is given in: C. Annemieke Romein et al., 'State of the Field: Digital History’, in: History, 105/365 (2020), 291-312 https://doi.org/10.1111/1468229X.12969. Piotrowski and Fafinski argue that quantative methods have been integrated in history for a long time: Michael Piotrowski and Mateusz Fafinski, 'Nothing New Under the Sun? Computational Humanities and the Methodology of History', Proceedings of the Workshop on Computational Humanities Research, Amsterdam 2020, https://serval.unil.ch/notice/ serval:BIB_907D09C8F5DF (2020-11-20).

16 Gerben Zaagsma, 'On Digital History', in: BMGN - Low Countries Historical Review, 128/4 (2013), 3-29.

17 For the concept of "digital hermeneutics" see: Marijn Koolen, Jasmijn van Gorp and Jacco van Ossenbruggen, 'Toward a Model for Digital Tool Criticism: Reflection as Integrative Practice', in: Digital Scholarship in the Humanities, 34/2 (2019), 368-85, https://doi.org/10.1093/llc/fqy048. Alberto Romele, Marta Severo and Paolo Furia, 'Digital Hermeneutics: From Interpreting with Machines to Interpretational Machines', in: AI \& SOCIETY, 35/1 (2020), 73-86, https://doi. org/10.1007/s00146-018-0856-2. See also Fickers, ,Update für die Hermeneutik‘.

18 Arguing with Digital History working group, Digital History \& Argument (Roy Rosenzweig Center for History and New Media, 13 November 2017), https://rrchnm.org/argument-whitepaper/ (2020-11-20). John Unsworth originally noted the Scholarly Primitives for the humanities: John Unsworth, 'Scholarly Primitives: What Methods Do Humanities Researchers Have in Common, and How Might Our Tools Reflect This?' presented at the Humanities Computing: 
- Selection: In addition to the processes of capturing and creating data, researchers identify the sources that provide relevant evidence and select them as their data. This selection is based on "the truthfulness of a source, its aesthetic qualities, its representativeness, or its uniqueness."

- Synthesis: In order to validate statements, methods, arguments, hypotheses or theories, the selected sources and any additionally created data are analysed for patterns and structures through different modes of composition and comparison, arranged according to time, place, topic or other ordering principles.

- Contextualisation: The creation of an accurate, reliable and persuasive account of the past requires a complex picture of an event, including its environment. The scale of this context may vary widely: from biographical to global history or from a microhistorical focus to perspectives spanning centuries.

- Communication: The representation of the results conveys all necessary information about the past and the argument, including a description of the processes of selection, synthesis and contextualisation that were undertaken.

A strategic and comprehensive approach to research data management includes measures to support these principles, such as enhancing selection possibilities by extended access to sources and to detailed descriptions of their features; building these principles into the research workflow with its data lifecycle.

A significant portion of historical source material will remain analogue research data. ${ }^{19}$ As Zaagsma notes, "The real challenge is to be consciously hybrid and to integrate 'traditional' and 'digital' approaches in a new practice of doing history". ${ }^{20}$ There is a danger, though, "that our narratives of history and identity might thin out to become based on only the most visible sources, places

formal methods, experimental practice, King's College, London 2000, http://www.people. virginia.edu/ jmu2m/Kings.5-00/primitives.html (2020-11-20).

19 According to the ENUMERATE 4 Survey, about 50 per cent of analogue heritage collections still need to be digitally reproduced, while only about 10 per cent are already digitised. Gerhard Jan Nauta, Wietske van den Heuvel, and Stephanie Teunisse, Report on ENUMERATE Core Survey 4, Europeana DSI 2- Access to Digital Resources of European Heritage, $31 \mathrm{Au}$ gust 2017, https://pro.europeana.eu/files/Europeana_Professional/Projects/Project_list/ ENUMERATE/deliverables/DSI-2_Deliverable\%20D4.4_Europeana_Report\%20on\%20ENUMERATE \%20Core\%20Survey\%204.pdf (2020-11-20), here 06.

20 Zaagsma, 'On Digital History', 17. 
and narratives". ${ }^{21}$ For this reason, a research data management strategy needs reflect hybrid approaches in mixed research data collections and analogue / digital combined methods. Also, the linkage between digital data and analogue sources has to be improved. The digitisation of heritage must be promoted through a systematic-programmatic strategy on the part of memory institutions and a research-driven effort.

In research data management, the data management plan (DMP) has become an established concept, and over the last decade funding bodies have increasingly advocated their use. ${ }^{22}$ DMPs may also become mandatory elements of research proposals in the humanities, even if at present few DMPs are created outside of larger collaborative project proposals. ${ }^{23}$ As a key instrument for research data management, the formal design has been already elaborated and adapted to the humanities domain. ${ }^{24}$

\section{The meaning of metadata}

Metadata describing analogue collections and sources - the catalogue of bibliographic records or inventory items, the oldest standard used by archives, libraries and museums to ensure the findability and accessibility of documents has long been essential to the historical method as a way of referencing sources.

21 Jennifer Edmond, 'Will Historians Ever Have Big Data?', in: Computational History and DataDriven Humanities, ed. by Bojan Bozic et al., Cham 2016, CDLXxxII, 91-105, here 11, https://doi. org/10.1007/978-3-319-46224-0_9.

22 Nicholas Smale et al., 'The History, Advocacy and Efficacy of Data Management Plans', in: BioRxiv, 2018, https://doi.org/10.1101/443499.

23 At present, in Germany there is rarely an obligation to submit a data management plan as part of the procedure for acquiring third-party funding. Nevertheless, a development in this direction is visible. For example, the German Research Foundation (DFG) explicitly requires an explanation in applications of how the research data used and generated in the project will be handled and demands that it be made available to the greatest extent possible. The application forms ask whether the project has prepared a data management plan. Due to this rapid development in the last five years, the obligatory submission of a DMP, as it has been common in EU-HORIZON funding for a long time, is not far away. https://web.archive.org/web/ 20201022005933/https://www.dfg.de/en/research_funding/proposal_review_decision/appli cants/research_data/index.html

24 Gisela Minn and Marina Lemaire, Forschungsdatenmanagement in den Geisteswissenschaften. Eine Planungshilfe für die Erarbeitung eines digitalen Forschungskonzepts und die Erstellung eines Datenmanagementplans, eSciences Working Papers 03, Trier 2017, http://nbn-resolving. de/urn:nbn:de:hbz:385-10715. 
Beyond transparent referencing, researchers add significant meta information to their sources by describing documents or objects on an item level that were previously only indexed on a collection level (or as an archival box). In addition to noting an item's specific attributes, an index of the content is created by assigning headwords, subject terms and categories; identifying entities; or providing abstracts. The scholarly principle of selection also draws relationships to other items within a collection and beyond it. The principle of contextualisation enables positioning items within topic-oriented, temporal or spatial scales. The relevance of these metadata is best expressed by Drucker, who notes that few other kinds of information have a "greater impact on the way we read, receive, search, access, use, and engage with the primary materials of humanities studies than the metadata structures that organise and present that knowledge in digital form". ${ }^{25}$ In sum, the scholarly principles of historical method, selection, synthesis and contextualisation, create rich metadata, which turns into research data in other contexts. Within the historically oriented humanities, a strict distinction between metadata and data would thus inevitably dissolve over time.

The diversity of research questions and approaches in the historically oriented humanities means there is no single metadata standard capable of describing all research data that is potentially relevant for historical research without forfeiting domain-specific information: thus, aiming for one common schema would be a mistake. Instead, a more decentralised approach promises to be more realistic and practicable. As long as metadata align with the standards of memory institutions, they can function as a knowledge organisation system about data, define minimum requirements for metainformation, enable automated exchange, foster international linking and allow for modular extension. ${ }^{26}$ In this framework, formal harmonisation to remove diversity is counterproductive but advancing the interoperability of existing resources and the different organisational principles adds significant value to institutional missions and research needs. A research data management strategy should pursue achieving interoperability at a higher level of abstraction by identifying and mapping common concepts and

25 Johanna Drucker, SpecLab: Digital Aesthetics and Projects in Speculative Computing, Chicago 2009, here 9.

26 This includes the metadata standards EAD (Encoded Archival Description) for archives, https://www.loc.gov/ead; LIDO (Lightweight Information Describing Objects), http://www. lido-schema.org/ and CIDOC-CRM (CIDOC Conceptual Reference Model), http://www.cidoccrm.org/ for museums, MARC: MAchine-Readable Cataloging (LoC), https://www.loc.gov/ marc/ for libraries, and METS/MODS: Metadata Encoding and Transmission Format / Metadata Object Description Schema, https://www.loc.gov/standards/mods/. 
entities in data and metadata. The use of a suitable existing standard as a starting point in a research project makes this mapping much easier. If necessary, scholars will extend them with regard to specific requirements of their historical methods and for describing and documenting particular kinds of research data, whether to account for historical perspectives that require defined temporal or spatial scales or, more complexly, to integrate models of uncertainty and change. ${ }^{27}$

As noted, historical metadata is often created to support an argument but also provides a high-quality indexing of the research data that is potentially useful for discovery. For the free access and reuse of research data, rich metadata that serves both intellectual reuse as well as improved retrievability is crucial. A research data management strategy for a community of practice in the historical sciences, that jointly develops concepts, the following concepts are especially important.

\section{Authority data}

Despite the widespread adoption of uniform principles of spelling and writing in the last century, the sources used by historical disciplines contain named entities rich in variants and dialects and, of course, extend across countless languages. Preserving these original variations is a fundamental prerequisite for historical understanding and source criticism; at the same time, their heterogeneity poses challenges to identify those variants that refer to the same concept. To work with them as research data, therefore, such variants must be additionally provided with identifiers to enable entity referencing and allow links through ontologies, multilingualism and Linked (Open) Data. ${ }^{28}$ In Germany, the Integrated Authority File (GND), as a standard for German-language authority data, mainly offers generalised concepts that are too broadly defined for scholarly applications in historically oriented fields. ${ }^{29}$ This is part of a wider issue: whereas specific

27 Andreas Kuczera, Thorsten Wübbena and Thomas Kollatz, Die Modellierung des Zweifels: Schlüsselideen und -konzepte zur graphbasierten Modellierung von Unsicherheiten, Sonderbände der ZfdG, Wolfenbüttel 2019, https://doi.org/10.17175/sb004.

28 Beat Estermann et al., Basisregister und kontrollierte Vokabulare als Wegbereiter für Linked Open Data in der Schweiz (Berner Fachhochschule, Institut Public Sector Transformation, 27 January 2020) https://arbor.bfh.ch/10249/ (2020-11-20).

29 Katrin Moeller, 'Standards für die Geschichtswissenschaft! Zu differenzierten Funktionen von Normdaten, Standards und Klassifikationen für die Geisteswissenschaften am Beispiel von Berufsklassifikationen', in: Aufklärungsforschung Digital. Konzepte, Methoden, Perspektiven, ed. by Jana Kittelmann and Anne Purschwitz, Halle 2019, 17-43, here 26-29. In the GND4C project, the German National Library is working with museums and archives to open the GND to cultural 
classifications or categories are required in the research process, indexing tools for memory institutions rely upon generic forms of description and mapping. Authority data therefore bridge dynamic linguistic variations and analytic vocabularies and ontologies. One can distinguish among universally accepted authoritydata standards (e.g. GND), subject-specific authority data used by larger communities and vocabularies and categories created by research projects to answer their research questions.

\section{Historical bibliographies}

Specialised or subject bibliographies continue to play a central role in the historically oriented humanities for referencing and researching literature. In digital form, these bibliographies are no longer closed but can potentially become open systems. Digital bibliographies often contain not only bibliographic records but also offer direct access to research literature. The bibliographically enhanced and high-quality metadata are also available as open data to other reference systems, such as union catalogues. ${ }^{30}$ The conception of research data as a qualified research output and as independent publications demands their integration in relevant specialised bibliographies and to link them to published research syntheses (i.e. monographs and articles). Combining traditional publications and research data in bibliographies will increase the recognition of data publications as respected scholarly achievements. Research syntheses will gain added value by being linked to the data on which they are based.

data from all sectors. See Detlev Balzer et al., 'Das Projekt “GND für Kulturdaten” (GND4C)', in: o-bib. Das offene Bibliotheksjournal / Herausgeber VDB, 6/4 (2019), 59-97, https://doi.org/10. 5282/o-bib/2019H4S59-97.

30 Eva Kraus and Matti Stöhr, 'On the Way to a "German Historical Bibliography”: Current State and Perspectives - The DFG Project "Continued Cooperative Development of Historical Subject Bibliographies”, in Historical Bibliography as an Essential Source for Historiography, ed. by Kristina Rexová et al. (Newcastle upon Tyre: Cambridge Scholars Publishing, 2015), 161-80. See also Wiebke Herr, Andreas C. Hofmann and Katrin Getschmann, 'Deutsche Historische Bibliografie. Präsentation, Partner und Perspektiven. Bericht über die Tagung am 25. Und 26. Oktober 2017 in München', in: Zeitschrift für Bibliothekswesen und Bibliographie, 65/2-3 (2018), 130-34, https://doi.org/10.3196/18642950186523168. 


\section{Ontologies}

In information science, an ontology is a shared, formalised, explicit and machineinterpretable representation of concepts (or terms) and their relationships to each other. ${ }^{31}$ Ontologies represent knowledge and enable the embedding of new insights into a knowledge system. Domain ontologies usually cover specific domains of knowledge and evolve independently of each other. The representation of ontologies is based on mathematical logic (description logics) and thereby language independent. By mapping the applied concepts to controlled vocabularies (authority data), they can be addressed via authority data identifiers. Identifiers not only help to distinguish ambiguous concepts from one another, but also allow concepts (and thus ontologies) to be maintained in several languages via labels. This is of great importance for the historically oriented disciplines, such as area studies or ancient history. At the same time, identifiers help to identify common concepts in different ontologies and thereby interconnect them. Linked ontologies form an important basis for knowledge graphs and Linked (Open) Data. They allow for a distributed development and maintenance of knowledge domains, while at the same time ensuring interconnectedness and interoperability.

\section{Knowledge graphs}

Knowledge graphs are based on ontologies. ${ }^{32}$ They supplement the concepts modelled in an ontology with references to concrete instances that represent these concepts, e.g. object records in archives, museums and research data repositories. ${ }^{33}$ Ontologies and knowledge graphs are thus suitable means for bringing together heterogeneous data sets and knowledge domains to make them interoperable. These characteristics meet the goal of linking research data across disciplines. Among the most relevant entities for the historically oriented humanities are: the representation of historical events; fuzzy/non-validated/contradictory facts; hypotheses; networks of people; and temporal, cultural and/or spatial variations in the meanings or labels of concepts.

31 Steffen Staab and Rudi Studer, Handbook on Ontologies, 2nd edn, Berlin 2009.

32 Amit Singhal, 'Introducing the Knowledge Graph: Things, Not Strings', in: Google Official Blog, 2012, https://blog.google/products/search/introducing-knowledge-graph-things-not/ (2020-11-20).

33 Sören Auer et al., 'Towards a Knowledge Graph for Science', in: Proceedings of the 8th International Conference on Web Intelligence, Mining and Semantics, 1-6, https://doi.org/10.1145/ 3227609.3227689. 
However, standardisation initiatives, and in particular ontological approaches and their technical implementation require significant efforts and pose several challenges:

- Formalising community-specific and research-driven description practices into quality-assured metadata standards, leading to discipline-specific extensions of established, proven standards by memory institutions;

- Enhancing the connectivity of research data through knowledge organisation systems with semantically clear and syntactically interoperable, machine-readable standards, enabling their integration in discovery systems;

- Developing technological solutions to enable the retrieval of data across information systems and metadata schemes, such as concrete implementations of ontological concepts and/or indexing layers;

- Integrating the use of metadata standards, quality assessment and data modelling into the basic skill sets of researchers and their methodology;

Furthermore, linked historical data presupposes a cultural change towards open data among researchers, their organisations and memory institutions through awareness raising, capacity building and innovation. But if common standards can be achieved, the reuse of data would also thrive across disciplinary boundaries.

\section{The challenges of open and FAIR data}

One of the motivations behind the FAIR principles is to enable the provision and reuse of data, a central aim of research data management. In the community of historically oriented humanities, we still see barriers to making data findable, accessible, interoperable and reusable. A commonly noted obstacle to the sharing of research data across all disciplines is the additional effort and resources necessary to prepare them. ${ }^{34}$ In the historically oriented humanities, however, the time-consuming preparation and analysis of the sources and research data is a given. In fact, the data types created during historical research and through the application of historical methods - e.g. validation, provenance, transcriptions, editions, entity extraction, contextualisation, registries, etc. - could directly improve the reuse potential of the sources in other research contexts.

34 Christine L. Borgman, 'The Conundrum of Sharing Research Data', in: Journal of the American Society for Information Science and Technology, 63/6 (2012), 1059-1078, https://doi.org/10. 1002/asi.22634. 
Thus, we are convinced that scholars do not shy away from data enrichment itself but rather from its publication and final revision. Why is this so? First, we think that a data publication culture has to be further established in our community. Second, we call for the specification of FAIR principles for the historically oriented humanities.

\section{Enabling a data sharing culture in the historically oriented humanities}

History remains one of the few humanities disciplines without domain-specific recommendations on research data management. This immediately suggests that a data sharing culture within the community has yet to be firmly established. Although we perceive a general awareness of the need for research data standards in the community, ${ }^{35}$ there still is a lack of common recommendations and guidelines on how to find and adhere to appropriate standards, workflows and services. We see a particular need for the community of researches, infrastructure services and memory institutions to work together on the improvement and establishment of a data sharing culture in the following five areas:

1) Systematic training in digital literacy provides researchers with the basic skills in information technology and research data management that enable them to enhance their workflows digitally, to gain skills in and perspectives on the analysis of digital data, and enrich their source criticism with digital methods.

2) A lack of knowledge about the use and application of metadata standards, taxonomies, controlled vocabularies and authority data results in a situation in which the enrichment and annotation of data and sources falls short of their potential. Those who provide the above mentioned knowledge organisation systems, such as archives and libraries, have to continue the process of opening these tools to researchers, who in turn have to become aware of their advantages.

3) A lack of institutional support leaves researchers detached from necessary research data management services. As temporary third-party funded projects are not able to compensate for the lack of institutionalised research data management support, different approaches towards capacity building, such

35 A general theme from the 4Memory Problem Stories, https://4memory.de/problem-storiesoverview/. 
as organised knowledge exchange, consulting services, recommendations for workflows and adaptable guidelines should be provided to the community.

4) Currently, publishing research data offers little professional reputation (e.g. in the context of granting tenure) and is not integrated into scholarly communication and quality management processes. A reputation economy for the historically oriented humanities requires continuous discussions towards a new data culture in our disciplines.

5) A lack of advice on legal and security issues hinder scholars in collecting research data and in subsequently publishing and reusing it. In addition to consultative support for researchers from their organisations, the community as a whole has to get involved in political discussions about copyright laws to achieve a regulatory framework that supports research.

\section{Discipline-specific FAIRness: The case for the historically oriented humanities}

Establishment of FAIR research data management will have to complement the efforts for changing the data sharing culture in the historically oriented humanities. The FAIR data principles themselves provide necessary guidelines for advancing data sharing and data-driven research. But as general recommendations, they have to be both adapted to specific issues and critically reflected upon within individual disciplines. Before making proposals for adapting each of the four principles to the historically oriented humanities, we would like to draw attention to two aspects that can reduce the FAIRness of research data in our community, especially in terms of accessibility and reusability.

First, copyright-related issues regarding humanities data arise quite often, as intellectual property rights are much more widespread than in the natural sciences, where machine-generated observational data are normally uncopyrighted. In our community, this poses a problem for the accessibility of research data and has to be dealt with by licensing of data in a meaningful way, e.g. through the family of Creative Commons licences. Still, uncertainty among researchers and a lack of advice remain common, and due to strict German and EU legislation, publication of research data remains difficult.

Second, considerations regarding privacy and research ethics have to influence our ideas about the FAIRness of research data in our community. For example, publishing interviews with people living in authoritarian or dictatorial regimes raises serious problems, since such publication could lead to negative consequences for them. Another example relates to research in small communities, in which 
despite anonymisation, people can be identified relatively easily. And even if anonymisation can be achieved, it might hinder reuse, as the full information of an interview will remain unavailable to other researchers. Thus, in the case of research involving people, data should be as open as possible but as closed as necessary, with the consequences that entails for their FAIRness.

The Global Indigenous Data Alliance has raised similar questions about the (re-)use of indigenous data by presenting the CARE principles for Indigenous Data Governance. ${ }^{36}$ These call for Indigenous data sovereignty, pledges for collective benefits from data ecosystems for Indigenous peoples and their authority to control data. They assert that researchers have special responsibilities when working with these data and should take the ethics of data collection into account in order to respect Indigenous peoples' rights and wellbeing. Although the development of the CARE principles was mainly supported by anthropologists, many aspects are relevant to historians as well and show how fruitful discipline-specific discussion of the FAIR principles can be. It is to this issue that we turn in the following section.

\section{Findability}

In historical research, the process of selection begins with finding primary sources. Therefore, reliable identification and registration of analogue artifacts is a longstanding practice in memory institutions. The use of archive, accession or inventory numbers as references is, similarly, a prevailing standard in historical research. However, many institutions and even established repositories still fail to provide persistent identifiers (PID) for their digital resources. Providers of relevant digital repositories and data collections should integrate PIDs where necessary and provide services to assign and resolve these. ${ }^{37}$ Persistent addressing should be performed not only on the level of data collections and research data sets, but also within, to be able to identify and find granular data as well.

In addition to ensuring the findability of data through identifiers and rich metadata, the discovery channels and search methods are also of major importance. Library catalogues and bibliographies play an important role in the historically oriented humanities. Thus, research data should be indexed in these

36 The Global Indigenous Data Alliance GIDA, 'CARE Principles of Indigenous Data Governance’, 2019, https://www.gida-global.org/care (2020-11-20); for an example see Tahu Kukutai and John Taylor (eds.), Indigenous Data Sovereignty: Toward an agenda, Acton 2016.

37 Such as DOIs (Digital Object Identifiers) provided by DataCite and URNs (Uniform Resource Name) provided by German National Library. 
traditional and domain-specific discovery tools alongside services such as Google Dataset Search or DataCite Search.

In the medium term, we see potential for creating a data space in which historians will be able to find multiple sources and linked data: material from archives, information on objects from museums, (scholarly) literature from libraries and research data from specialised repositories. This would increase the connectivity among the main scholarly infrastructures, overcoming a fragmentation of scholarly records that leaves a digitised source at the archive, its transcription in a repository and its reference in a monograph at the library by enabling the contextualisation of the data that is essential to the historical method. Technologies like authority data identifiers, ontologies and knowledge graphs are a promising way towards this path.

While cataloguing builds upon established heuristic search practices, concepts like the knowledge graph introduce new retrieval methods and differ from traditional browsing and searching for sources in memory institutions' information systems. As a consequence, and to ensure findability, the heuristics for datasets (and their different logics) have to be integrated into historical methodology and become integral parts of the professional training for historians and other scholars.

\section{Accessibility}

The FAIR principles define accessibility primarily through technical protocols relevant for service providers but provide no recommendations to researchers about how to improve accessibility beyond physical access to files. Filling this gap is left to the disciplines, which need a broad and open debate about the specific implications that data accessibility has for them. Transparency and reproducibility are essential quality requirements that are shared by the FAIR principles and the historical method. ${ }^{38}$ Assessing and assuring these requirements, though, differs significantly between quantitative and qualitative research methods. Even if the scholarly principles of evidence selection, synthesis and contextualisation are described, comprehending, modelling and understanding hermeneutic processes is challenging. If guidelines for such documentation, models of scholarly

38 Klaus E. Müller and Jörn Rüsen, Historische Sinnbildung. Problemstellungen, Zeitkonzepte, Wahrnehmungshorizonte, Darstellungsstrategien, Reinbek 1997. See also Fickers, ,Update für die Hermeneutik', 2020. 
practice and rule-based procedures are available, it becomes easier to make the interpretive steps of a research project comprehensible, even replicable.

Explaining the selection of sources and methods is already an established practice in historical research. Documentation within data publication will provide more than a general understanding of the research process. ${ }^{39}$ It is an important instrument for understanding the composition, creation and archiving of data in the humanities, even enabling reproducible workflows. ${ }^{40}$ In addition to data curation, accompanying processes in the sense of "data publishers" are essential for the development of institutional support services for the humanities.

Ensuring accessibility also includes technical access through standardised, open and processable file formats. It is not sustainable to store texts or data in containers that are difficult to reuse, as would be the case with text modules in image files or PDFs. Prioritising accessibility, to the contrary, encourages selecting and using formats that can be archived over the long term and still remain accessible and reusable. ${ }^{41}$ Accessibility does not require the data to be openly available. Even if the data itself must be blocked or deleted due to data protection or to ethical or licensing restrictions, metadata and documentation can provide accessible information about it.

\section{Interoperability}

Establishing technical interoperability is a complex, but also an already welldefined task. It is more difficult to ensure the interoperability of the content: here, workflows and standards are still lacking, especially in historical research. Memory institutions, though, excel at the prerequisites of interoperability: precise

39 Methods from the social sciences, for example, provide orientation here: Jan-Ocko Heuer et al., 'Kontextualisierung qualitativer Forschungsdaten für die Nachnutzung: eine Handreichung für Forschende zur Erstellung eines Studienreports', Bremen 2020, https://doi.org/10. 26092/elib/166.

40 Jonathan Blumtritt and Patrick Sahle, Forschungsdatenmanagement als Puzzlespiel: Institutionelle Aufgaben und Rollen bei der Versorgung der Geistes- und Kulturwissenschaften, in: Archivar, 73/1 (2020), 19-24, here 21. See also DHd AG Datenzentren, Geisteswissenschaftliche Datenzentren im deutschsprachigen Raum - Grundsatzpapier zur Sicherung der langfristigen Verfügbarkeit von Forschungsdaten (Zenodo, 3 January 2018), https://doi.org/10.5281/zenodo. 1134760.

41 This not only has advantages for searchability and retrievability but also facilitates processes of interoperability and reuse. Kyle Rimkus et al., 'Digital Preservation File Format Policies of ARL Member Libraries: An Analysis', in: D-Lib Magazine, 20/3-4 (2014), https://doi.org/ 10.1045/march2014-rimkus. 
descriptions, persistence and authority data. Historical research, on the other hand, thrives on dynamic rearrangements, differentiated knowledge systems and accounting for multiple perspectives. To unleash its creative potential and reach significant conclusions, humanities research addresses the variety of contrasting perspectives that critical reconsiderations of knowledge production have stressed, particularly in the wake of long-running historiographical debates and methodological disputes. Making information interoperable means interlinking systems that require stable persistence (and metadata) with the dynamic multiperspectivity of research data.

The key problem relates to the comparability of terms, objects and scope. For the historical disciplines, similarities and differences across time are central issues, which require description through the categories, characteristics and properties of entities in a time-related, contextualised and conceptualised manner. Spatial, object and personal data - as well as entities of social stratification and relationship - also play central roles. For this purpose, controlled vocabularies, authority data, the GND and the setting of standards are important prerequisites: interoperable metadata, taxonomies or ontologies can be built on them. But the temporal and cultural binding of terms is often related to specific entities, and therefore, the historical and cultural perspectives have to be integrated in authority data or added as their extension. This may also encourage a broader use of vocabularies and reference systems. ${ }^{42}$

Historical disciplines have yet to have a comprehensive discussion about which common authority data and standards should be developed or established. Moreover, multilingualism has to be taken into account and is addressed through bridging national and international standards, e.g. the Virtual International Authority File (VIAF) or the Historical International Classification of Occupations (HISCO), which interlinks existing national authorities. In the end, the implementation of interoperability through authority data and standards will require creating data curation tools which either enhance or automate the data enrichment, in order to ease the process for the researchers.

42 Torsten Hiltmann, 'Forschungsdaten in der (digitalen) Geschichtswissenschaft. Warum sie wichtig sind und wir gemeinsame Standards brauchen', in: Digitale Geschichtswissenschaft (Blog), https://digigw.hypotheses.org/2622 (2020-11-20). See also Moeller, ,Standards für die Geschichtswissenschaft!‘, 17-22. 


\section{Reusability}

The documentation of data quality is essential for other researchers to assess its value for further research. It has to go beyond minimal metadata like title or author, as these are not sufficient to document the historical principles of selection, synthesis and contextualisation. For the historically oriented humanities the concept of provenance is crucial, be it an ancient document from an archive or a born digital data set resulting from prosopographic research. A digital source criticism asks for information on the origin of data objects, their genesis and the modifications to which they were subjected during the research process.

While not all data necessarily requires rich annotation, extensive documentation or flawlessness in order to be reused, it is vital that the quality levels of data are made transparent to others. Defining different quality requirements and levels could provide a basic, but immediate and structured assessment of the data quality. These requirements and levels of data quality could be described and referenced externally, in a similar way to the Creative Commons licences. Scalable quality criteria could be implemented, allowing different degrees of data quality as sufficient according to the research settings. Otherwise, we are afraid that emphasis on data quality and documentation alone could prevent scholars from publishing data at all. ${ }^{43}$

Reusability as a scholarly practice means not only that the relevant information is stored in metadata but also that the community knows, how to cite data sets and whom to attribute, issues that are more complicated in huge projects where the people involved fulfill different roles and functions. Historians are used to working with sources that are centuries or even millennia old. So what does reuse mean in the long term preservation of born-digital research data? In contrast to the ten years that are proposed by the DFG and that may be sufficient in some disciplines, we are convinced that a much longer perspective is needed, one closer to the practices of archives and libraries which span centuries, even if we are aware of the huge and partly unsolved technical and technological challenges that accompany such an approach. And for scholarship in the humanities, we might even question whether reuse is the proper term at all. But can we speak of a hermeneutic reuse? Should we not rather call for spelling the R in FAIR as reinterpretable? Could research data serve as a source of the reasoning and hermeneutics of humanities scholars? Finally, within the technological concepts of

43 Eva Schlotheuber and Johannes Paulmann, 'Digitale Wissensordnung und Datenqualität: Herausforderungen, Anforderungen und Beitrag historisch arbeitender Wissenschaften', in: Archivar, 73/1 (2020), 9-12, here 11. 
harmonisation and interoperability relevant for FAIRness, humanities based concepts and priorities such as diversity, multilinguality, historical context, and complexity have to be preserved and introduced into computational and algorithm based approaches in order to avoid the "risk of losing thick description" 44 and a "future of record scarcity". ${ }^{45}$

\section{A call to action}

The historically oriented humanities are at a moment of transition of their research practices, their research objects and the organisational, economic and societal conditions of historical research. Even if the digital transformation has been overwhelming, it remains the case that the answer from the research communities cannot be passivity, as Gehring notes in a call for engagement from the historically oriented humanities to participate in the debates and developments in science policies, information infrastructures and research methods. ${ }^{46}$ Data is meeting history (and the historically oriented humanities), and this will be more than a fleeting acquaintance: In many ways, digital data will be at the core of historiography. For this to become an equal and enduringly stable relationship, a discipline-specific research data management strategy and the adaptation of the FAIR principles to the scholarly principles of historical research appear to be reliable building blocks. A promising mode of participation has also emerged: a self-organised and collaborative coalition of research, memory and information infrastructure institutions.

44 Erzsébet Tóth-Czifra, 'The Risk of Losing Thick Description: Data Management Challenges Arts and Humanities Face in the Evolving FAIR Data Ecosystem', in: Digital Technology and the Practices of Humanities Research, Cambridge 2019, 235-266, https://doi.org/10.11647/OBP.0192.

45 Roy Rosenzweig, 'Scarcity or Abundance? Preserving the Past in a Digital Era', in: The American Historical Review, 108/3 (2003), 735-62, https://doi.org/10.1086/ahr/108.3.735.

46 Petra Gehring, 'Digitalität als Sache von fachlichem Unterscheidungs- und Entscheidungsbedarf. Hype oder Chance? Experiment oder Falle?', in: Die Geschichtswissenschaft im Digitalen Zeitalter, VHD Journal, 9 (2020), 22-25, here 24. 